H I G H L I G H TS

EVO-DEVO

\section{Regulatory affairs}

A crucial question in evo-devo research is how alterations in gene function result in morphological variation. Plant and animal studies have indicated that the evolution of morphological structures might be caused by mutations in the control regions of regulatory genes, such as transcription factors and their targets. A recent study by Grandien and Sommer lends support to this idea by showing that two orthologous Hox genes in two related nematode species are functionally equivalent, despite having quite divergent coding sequences and clearly distinct developmental roles in the two species.

Nematodes are well suited to comparative morphological studies, as homologous cellular and developmental processes are easily detected in closely related species. A classic example is the development of the nematode vulva - the mating and egglaying structure - in Caenorhabditis elegans and Pristionchus pacificus. The C. elegans vulva develops from 12 epidermal cells: six form the vulval equivalence group, and descendents of only three of them differentiate into the vulva proper. The Hox gene lin-39 is required for two steps in vulval development: to establish the vulval equivalence group and, later, for vulval differentiation. Things are quite different in P. pacificus: for example, the vulval equivalence group consists only of three cells; in addition, those cells that do not differentiate into the vulva die, unlike in C. elegans, in which they fuse with the surrounding tissue. The func-

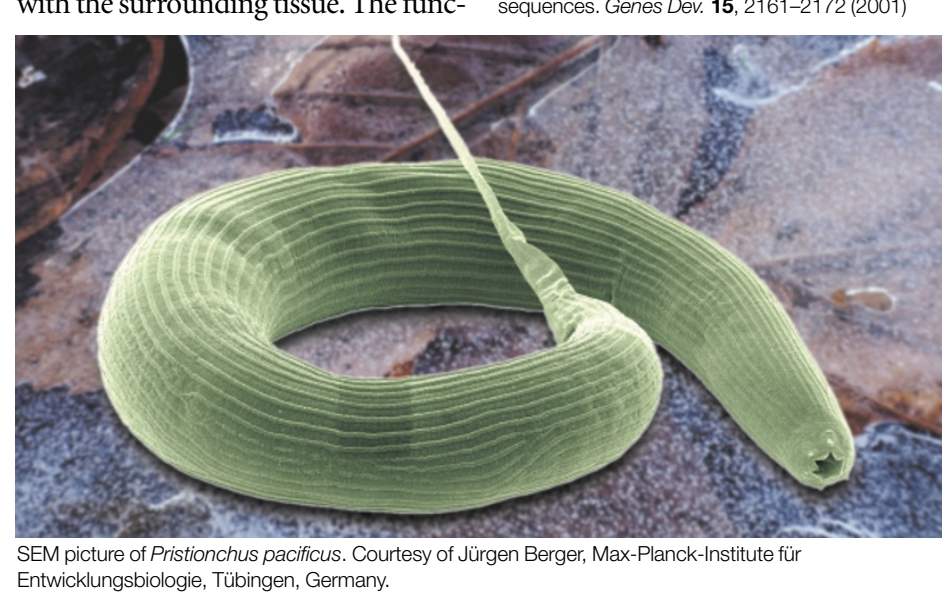

Entwicklungsbiologie, Tübingen, Germany. tion of lin-39also differs in P. pacificus - the gene is required to prevent cells required later for vulval formation.

What is the genetic basis for the different functions of LIN-39? A predicted MAPK docking and phosphorylation site at the carboxyl terminus of C. elegans ( $\mathrm{Cel}$ ) LIN-39, which is absent from the P. pacificus (Ppa) LIN39 , at first seemed like a convincing candidate, but it was ruled out by functional studies. The lack of any other obvious structural differences between the two LIN-39 proteins gave the first hint that they might be functionally equivalent - a possibility the authors confirmed by transgenic studies. Despite the limited amino-acid similarity between the two proteins, a transgene that contains the Ppa-lin-39 cDNA was found to rescue the egg-laying and vulval cell-lineage defects of a Cel-lin-39 mutant but, crucially, only when driven by Cel-lin-39 control regions. The same transgene also restored the presence of the vulval cell neurons, which require lin-39. The conclusion seems to be that the key to the difference between the two proteins lies in their respective control regions.

Follow-up studies will fill in the details, but the message from this paper is that developmental processes can evolve more readily through changes in gene expression than through tinkering with protein function.

Tanita Casci

\section{Di) References and links} ORIGINAL RESEARCH PAPER Grandien, K. \& Sommer, R. J. Functional comparison of the nematode Hox gene lin-39 in C. elegans and $P$. pacificus reveals evolutionary conservation of protein function despite divergence of primary sequences. Genes Dev. 15, 2161-2172 (2001) from undergoing apoptosis, but is not

\section{IN BRIEF}

\section{TECHNOLOGY}

Identification of a gene associated with Bt resistance in Heliothis virescens.

Gahan, L. J. et al. Science 293, 857-860 (2001)

Bt toxin resistance from loss of a putative carbohydrate-modifying enzyme.

Grififtts, J. S. et al. Science 293, 860-864 (2001)

The expression of the Bacillus thuringiensis (Bt) toxin in transgenic plants has become an important strategy for pest control in many plant species. However, its use is threatened by the development of pest resistance to Bt toxin. Although resistance is not yet widespread in the field, resistant mutants of several species have been identified in the laboratory. In these two studies, the genetic basis for toxin resistance has been defined in the tobacco mudworm and the nematode Caenorhabditis elegans: mutations in both have been found that could affect the binding and absorption of the toxin in the gut. These mutations provide valuable insight into the mechanisms of Bt toxin resistance and will allow studies of how best to manage the development of toxin resistance in the field.

\section{EVOLUTION}

Recent origin of Plasmodium falciparum from a single progenitor.

Volkman, S. K. et al. Science 293, 482-484 (2001)

Opinions are divided on the origins of a malaria parasite, Plasmodium falciparum. Abundant genetic variation at certain loci indicates an ancient origin, whereas low variation at silent sites in coding sequences indicates a recent origin. By analysing 25 intron sequences of general metabolic and housekeeping genes, Volkman and colleagues found very few single nucleotide polymorphisms and estimated that $P$. falciparum originated from 9,500 to 23,000 years ago. This is consistent with a recent finding that malaria has had a major impact on human evolution only since the introduction of agriculture, $\sim 10,000$ years ago (see In Briefs, August 2001).

\section{POPULATION GENETICS}

Are rare variants responsible for susceptibility to complex diseases?

Pritchard, J. K. Am. J. Hum. Genet. 69, 124-137 (2001)

It is assumed that alleles underlying common diseases are old and therefore common in the population (the "common disease, common variant" hypothesis). By incorporating mutation, random genetic drift and purifying selection into a mathematical model for how complex disease loci evolve, this paper shows that, although there might be many susceptibility alleles, there is likely to be extensive allelic heterogeneity at many of these loci. This result has implications, among others, for the effectiveness of association mapping, which depends on rare susceptibility variants. 